\title{
Hospital de Ensino promovendo a saúde no território*
}

Teaching Hospital: promoting health in the territory

Hospital de Enseñanza promoviendo la salud en el territorio

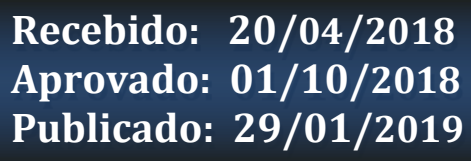

Recebido: 20/04/2018

Aprovado: 01/10/2018

Publicado: 29/01/2019

\author{
Nakita Maria Komori ${ }^{1}$ \\ Luiz Antonio Pertili Rodrigues de Resende ${ }^{2}$ \\ Dalmo Correia Filho ${ }^{3}$ \\ Mirtes Helena dos Reis ${ }^{4}$
}

O presente estudo tem como objetivo relatar a experiência de acadêmicos de enfermagem integrantes do Programa de Educação Tutorial — Enfermagem em atividade educativa extramuros proposta por um Hospital Universitário Federal. A atividade de extensão proposta foi realizada em dois encontros entre os meses de outubro e dezembro de 2016 em duas comunidades na cidade de Uberaba. Como estratégia de aproximação e interação com a população foram utilizados aferição de pressão arterial e glicemia rápida e teste de hepatite $C$, além de confecção de materiais lúdicos, como cartazes, mostruários de remédios, dinâmicas, panfletos, gravuras, instrumentos de anotação das orientações e outros meios para interação com o público. Os temas abordados nessa experiência foram: diabetes; hipertensão arterial; hepatites; uso correto de medicamentos; interpretação de rótulos de produtos alimentares industrializados; eletricidade no domicílio; reciclagem de resíduo doméstico. A atividade permitiu aos discentes de enfermagem uma oportunidade de interagir com discentes e profissionais de outros cursos da área da saúde, e dessa forma, ter uma visão interdisciplinar sobre temas de grande relevância para a promoção da saúde da população, além de uma maior proximidade e interação dialógica com a comunidade. Descritores: Educação em saúde; Saúde pública; Hospitais de ensino, Serviços de integração docente-assistencial.

This study aims to report the experience of undergraduate nursing student's members of the Programa de Educação Tutorial of the Nursing course (Program of Tutorial Education - PET Nursing) in an educational extramural activity proposed by a Federal Teaching Hospital. The extension activity proposed was conducted in two meetings from October to December 2016, in two communities in the city of Uberaba, MG, Brazil. As a strategy to approach and interact with the population, arterial pressure and rapid glycemic measures, as well as hepatitis$\mathrm{C}$ tests were used, in addition to the confection of ludic materials, such as banners, medication showcases, leaflets, drawings, tools to write down the guidance received and other means of interaction with the public. The themes addressed by this experience were: diabetes; arterial hypertension; hepatitis; the correct use of medication; interpretation of industrialized food product labels; electricity in the residence; recycling of domestic waste. The activity gave the students an opportunity to interact with professors and professionals from other health field courses, and therefore, to have an interdisciplinary view on highly relevant themes to promote the health of the population, in addition to being closer and having a dialogic interaction with the community.

Descriptors: Health education; Public health; Hospitals teaching; Teaching care integration services.

El presente estudio tiene como objetivo relatar la experiencia de académicos de enfermería integrantes del Programa de Educación Tutorial - Enfermería en actividad educativa extramuros propuesta por un Hospital Universitario Federal. La actividad de extensión propuesta fue realizada en dos encuentros entre los meses de octubre y diciembre de 2016 en dos comunidades en la ciudad de Uberaba, MG, Brasil. Como estrategia para la aproximación e interacción con la población fue utilizada la medición de presión arterial, glucemia rápida y test de hepatitis C, además de confección de materiales lúdicos, como carteles, muestras de remedios, dinámicas, panfletos, grabados, instrumentos de anotación de las orientaciones y otros medios para interacción con el público. Los temas abordados en esta experiencia fueron: diabetes; hipertensión arterial; hepatitis; uso correcto de medicamentos; interpretación de rótulos de productos alimentarios industrializados; electricidad en el domicilio; reciclaje de residuo doméstico. La actividad permitió a los estudiantes de enfermería una oportunidad de interactuar con alumnos y profesionales de otras carreras del área de salud y, de esta forma, tener una visión interdisciplinaria sobre temas de gran relevancia para la promoción de la salud de la población, además de una mayor proximidad e interacción dialógica con la comunidad.

Descriptores: Educación en salud; Salud pública; Hospitales de enseñanza; Servicios de integración docente asistencial.

${ }^{1}$ Acadêmica em Enfermagem da Universidade Federal do Triângulo Mineiro (UFTM), Uberaba, MG, Brasil. ORCID: 0000-0002-5694-3417 E-mail: nakitakomori@hotmail.com

2. Médico. Especialista em Gestão de Hospitais Universitários no SUS. Mestre em Medicina Tropical e Infectologia. Professor da UFTM, Uberaba, MG, Brasil. ORCID: 0000-0001-6276-7248 E-mail: luizantonio@mednet.com.br

3. Médico. Especialista em Infectologia. Mestre em Medicina Tropical. Doutor em Infectologia e Medicina Tropical. Professor Associado IV da Disciplina de Doenças Infecciosas e Parasitárias e Gerente de Ensino e Pesquisa do Hospital de Clínicas da UFTM, Uberaba, MG, Brasil. ORCID: 0000-0002-2174-5058 E-mail: dalmo@mednet.com.br

4.Técnica de Enfermagem. Técnica em Enucleação. UFTM, Uberaba, MG, Brasil. ORCID: 0000-0002-3651-5503 E-mail: mirtes.reis@uftm.edu.br * Fonte de Financiamento - Secretaria de Educação Superior do Ministério da Educação/SESU-MEC. 


\section{INTRODUÇÃO}

A s novas formas de organização dos serviços e as mudanças ocorridas no sistema de saúde, bem como as descobertas científicas e o desenvolvimento de tecnologias cada vez mais complexas são fatores que influenciam as mudanças na formação do enfermeiro. Essas transformações requerem que os profissionais da saúde tenham maior subsídio teórico e principalmente prático para realizarem suas funções com responsabilidade ${ }^{1}$.

Por sua vez, as ações de educação em saúde apresentam grande relevância no processo de formação do enfermeiro, visando ampliações na concepção teórico-prática, em especial quando o usuário é o eixo central, o que proporciona espaços que permitam sua participação no contexto da saúde ${ }^{2}$.

De forma geral, o usuário e seus aspectos ainda são tratados como um objeto de estudo fragmentado e não como um coletivo ${ }^{3}$. Apesar das informações oferecidas para as comunidades, essas não são suficientemente efetivas em relação à saúde. A educação em saúde é uma forma de integrar esse indivíduo na participação popular e nos serviços de saúde ${ }^{4}$.

Historicamente os hospitais, com seus aparatos tecnológicos e político-legal têm se constituído como um ambiente para tratamento e cura, limitando o espaço da clínica à cura ou reabilitação, distanciando-a da promoção da saúde. Os modelos de gestão e cuidado dessas instituições tem dificultado a construção de espaços de autonomia e a participação daqueles que buscam atenção à saúde, os quais poderiam contribuir para efetivação de uma prática promotora da saúde ${ }^{1}$.

Neste sentido, a responsabilidade na promoção da saúde tem sido direcionada ao setor de atenção primária; no entanto, é sabido que os hospitais são parte importante da comunidade e que toda instituição, especialmente aquelas envolvidas com o serviço público, deve ser ativamente comprometida com o planejamento para a promoção da saúde. Alguns países desenvolvidos têm implementado políticas no intuito de orientar as instituições de saúde para a saúde da comunidade, educação continuada e capacitação comunitária ${ }^{5}$.

Em 2004, o Ministérios da Saúde e o Ministério da Educação estabeleceram critérios para certificação dos hospitais universitários, visando sua missão acadêmica, assistência e relação com a rede pública de saúde (portaria conjunta no. 1000 de 15 de abril de 2004) além da criação do Programa de Reestruturação dos Hospitais de Ensino no âmbito do SUS (portaria $\mathrm{n}^{\circ} 1702$ BRASIL, Ministério da Saúde, 2004) ${ }^{6}$.

Em 2012, foi criada pela Lei 12.550 a Rede Brasileira de Serviços Hospitalares EBSERH -, com a proposta de modernização do modelo de gestão dos Hospitais Universitários. Desde então, a rede EBSERH tem provocado seus hospitais a promoverem ações de saúde junto à comunidade, seja no aspecto da intervenção quanto na promoção da saúde. Em especial no Triângulo Mineiro, o HC-UFTM tem constantemente enfrentado questões relacionadas as mudanças de paradigma, seja no modelo de gestão, de cuidado, ou acadêmico.

O HC-UFTM tem promovido ações para fortalecer a integração de docentes, técnicos e discentes (graduação e pós-graduação), sendo uma dessas ações a educação em saúde, como ferramenta que permite a disseminação de conhecimento nas populações. Ainda mais quando são utilizadas técnicas facilitadoras de construção coletiva dos saberes, favorecendo um espaço de relações dialógicas, participativas e afetivas que possam esclarecer dúvidas, compartilhar aprendizados e gerar a adoção novas medidas de saúde frente aos assuntos abordados ${ }^{7}$.

Ademais, vê-se a necessidade de aproximação do profissional de saúde, em especial aqueles dos serviços terciários, com a realidade e a prática vivenciada pelo seu usuário, visando a transformação do perfil dos futuros profissionais de saúde ${ }^{1}$.

Durante a graduação de enfermagem, os alunos são incentivados a realizarem práticas educativas em serviços de saúde, como salas de espera de Unidades Básicas de Saúde e projetos educativos no ensino fundamental. Contudo não é incentivada a pratica educativa 
em saúde na comunidade com o envolvimento de profissionais de saúde do nível terciário, aspecto desafiador devido ao comprometimento necessário ${ }^{8}$.

No intuito de implementar práticas educativas em saúde, em espaços que ultrapassem os muros do serviço de saúde, busca-se fortalecer a formação desses profissionais, em especial o enfermeiro. Discute-se na atualidade sobre iniciativas que passam a apoiar essa construção ${ }^{9}$.

O Programa de Educação Tutorial (PET) foi criado em 1979 com o nome de Programa Especial de Treinamentos implementado pela fundação Coordenação de Aperfeiçoamento de Pessoal de Nível Superior (CAPES), com intuito de formar grupos de estudo de alunos bolsistas ${ }^{10}$.

No ano de 2005 o PET é intitulado Programa de Educação Tutorial, cujo objetivo é desenvolver atividades de ensino, pesquisa e extensão como forma de implementar as Políticas Públicas e desenvolvê-las em sua área de atuação ${ }^{11}$. Nesta direção, tem-se no curso de graduação em enfermagem da UFTM o PET pautado nas políticas públicas de saúde, que contribuem com a formação ampliada e horizontalizada do profissional enfermeiro, voltada à promoção da saúde.

0 presente estudo tem como objetivo relatar a experiência de acadêmicos de enfermagem integrantes do Programa de Educação Tutorial - Enfermagem em atividade educativa extramuros proposta por um Hospital Universitário Federal. Isto como unidade integradora do ensino e serviço, com missão voltada para a formação do enfermeiro e demais profissões.

\section{MÉTODO}

Trata-se de um relato de experiência dos acadêmicos integrantes do PET Enfermagem em atividade educativa extramuros proposta pelo Hospital de Clínicas da Universidade Federal do Triângulo Mineiro (HC-UFTM). Essa atividade de extensão, denominada "HC nos Bairros", foi desenvolvida com a participação integrada dos Programas de Educação Tutorial da Enfermagem e Medicina, ligas acadêmicas, programas de residência, técnicos administrativos do HC-UFTM e docentes da universidade junto as comunidades do município de Uberaba.

As ações foram realizadas no período de outubro a dezembro de 2016 em dois sábados no período matutino, em locais previamente pactuados entre os coordenadores do projeto e representantes da comunidade, esclarecendo os motivos das atividades de educação em saúde pretendidas.

Em reuniões de planejamento com os envolvidos no projeto, definiu-se as seguintes estratégias para aproximação e interação com a população: aferição de pressão arterial; teste de glicemia rápida; teste de hepatite $\mathrm{C}$; e a elaboração de materiais lúdicos como: cartazes, mostruários de remédios, dinâmicas, panfletos, gravuras, instrumentos de anotação das orientações, entre outros meios, buscando promover interações positivas e educação em saúde com o público.

Os temas abordados nessa experiência foram: diabetes, hipertensão arterial, alimentação saudável, tabagismo, fatores de risco para problemas cardiovasculares, hepatites, uso correto de medicamentos, interpretação de rótulos de produtos alimentares industrializados, eletricidade no domicilio e reciclagem de resíduos domésticos.

Utilizou-se como arcabouço teórico para esse relato de experiência o "agir comunicativo" definido por Habermas como uma diferenciação entre os mundos objetivo, social e subjetivo num paradigma legitimador do discurso e da ação ${ }^{12}$.

\section{RESULTADOS}

Para o planejamento das ações com a comunidade, os discentes do grupo PET Enfermagem juntamente com os demais envolvidos, Pet Medicina, ligas acadêmicas e profissionais do HC-UFTM, contribuíram de forma efetiva para definição dos temas, das estratégias, bem como na elaboração de materiais lúdicos como cartazes, mostruários de remédios, dinâmicas, panfletos, gravuras, instrumentos de anotação das orientações, e outros meios para interação com o público.

Foi possível perceber, durante $o$ planejamento da ação, uma maior e melhor interação social dos envolvidos, em especial 
os alunos do PET Enfermagem, contribuindo para que o grupo desenvolvesse um trabalho interdisciplinar com vistas à promoção da saúde e prevenção de doenças, com a participação popular por meio de projetos fora do ambiente hospitalar.

Quanto ao primeiro encontro, realizado no Centro Comunitário da área de abrangência, os integrantes do grupo PET participaram da realização dos testes glicêmico e Hepatite $\mathrm{C}$, como estratégia de aproximação com a população, em que foi possível manter uma relação dialógica com a população a respeito do diabetes, hipertensão arterial e alimentação saudável, a partir do uso de cartazes e dinâmicas como painel de perguntas e respostas sobre os temas.

No segundo encontro, realizado em um lar de caridade, também foi ofertada a aferição de pressão arterial e teste glicêmico como estratégia para aproximação e interação com a população. Nesse dia, os integrantes do PET Enfermagem realizaram ação de educação em saúde sobre a dengue e o uso correto de medicação.

Foram elaborados cartazes sobre os principais aspectos da dengue como prevenção, diagnóstico e tratamento, bem como sobre alguns dos medicamentos utilizados no dia a dia das pessoas. Realizouse também uma dinâmica em que os participantes sorteavam perguntas, e de forma interativa e lúdica, as respostas foram apresentadas pelos integrantes do Pet Enfermagem.

Sempre que foram necessárias orientações mais específicas à população, os integrantes do PET contavam com a orientação e apoio dos profissionais e professores responsáveis pela ação.

Essa atividade de extensão permitiu aos integrantes do Pet Enfermagem uma oportunidade para interação e integração com acadêmicos e professores de outros cursos da área da saúde, com profissionais de um hospital de ensino e com a comunidade.

\section{DISCUSSÃO}

De forma geral, são escassas as publicações científicas sobre experiências que promovam integração entre serviços de saúde e comunidade a partir de ações que ultrapassem os muros do serviço de saúde. Essas ações podem contribuir para melhorar o acesso e transformar as práticas e cuidados de saúde para as populações ${ }^{8}$.

Em muito, estratégias dessa natureza proporcionam subsídios para que o indivíduo compreenda e transforme sua realidade, possibilitando que transitem de uma consciência ingênua para um olhar crítico, e gerando reflexão em relação ao cuidado com sua saúde ${ }^{13}$.

A comunicação é um dos meios para fazer educação em saúde, pois ela promove a interação entre os seres humanos de uma maneira menos formal. Porém, observa-se barreiras que dificultam essa comunicação entre o profissional da área da saúde e o usuário, devido à diferença na linguagem e saberes, além das limitações inerentes tanto do receptor quanto do transmissor ${ }^{14}$.

Aspectos que facilitam ações educativas contextualizadas aos cenários comunitários relacionam-se à utilização de abordagens e técnicas que façam aproximações de linguagem e facilitem a compreensão dos temas como a utilização de materiais lúdicos, e que estejam organizadas de maneira horizontalizada, ampliando os passos para os diálogos e trocas conjuntas ${ }^{15}$.

Sobretudo, implementar ações que envolvam a dialogia e o agir comunicativo junto a comunidades é desafiante para graduandos de enfermagem. As intervenções educativas extramuros dos serviços de saúde devem ser estimuladas na graduação, como uma estratégia proveitosa para uma formação que esteja inclinada à resolução de problemas contemporâneos de saúde ${ }^{16}$.

Dessa forma, o cidadão é posto no centro das ações de educação em saúde como participante vivo, pensante, comunicante, no contexto da comunidade, levando a uma relação dialógica ente os sujeitos profissionais de saúde e população ${ }^{18}$.

Esse fato permite inferir a necessidade de se reduzir a distância de processos educativos em saúde, estabelecendo uma relação mútua do fazer organizacional dos serviços de saúde; assim, estes tipos de atividades tem mostrado melhor 
aproveitamento, pois proporcionam aprendizagem sobre o tema, estimulam a compreensão e reflexão sobre o assunto de forma prazerosa, e a formação de relações entre o conhecimento proporcionado pelo lúdico e a realidade vivenciada.

Nessa direção, o diálogo aberto e responsável, proporcionado pelas ações de educação, é um dos caminhos possíveis para alcançar as boas práticas de saúde. O Agir Comunicativo proposto por Habermas, alcança, assim, a instrumentalização das relações e as boas práticas de promoção da saúde ${ }^{19}$.

\section{CONCLUSÃO}

Essa vivência permitiu aos envolvidos observar a importância do diálogo como forma de aprendizagem na educação em saúde, além de oferecer oportunidades de se trabalhar a comunicação, como estratégia para educação em saúde.

Esta proposta de olhar dialógico com a comunidade, considerando especialmente aspectos como: a realidade do processo de saúde e doença, perspectivas da prevenção de doenças e promoção da saúde, trouxe ao grupo a reflexão acerca do preparo para esse tipo de atividade, bem como da relevância para a formação do profissional.

É possível que a inclusão de profissionais de nível terciário atuando em conjunto com a atenção primária à saúde tenha lacunas em termos de pesquisa, o que traz à tona a contribuição desta experiência.

\section{REFERÊNCIAS}

1. Trevisan DD, Minzon DT, Testi CV, Ramos NA, Carmona EV, Silva EM. Formação de enfermeiros: distanciamento entre a graduação e a prática profissional. Ciênc Cuid Saúde [Internet]. 2013 [citado em 13 set 2017]; 12(2):331-7.

DOI:

http://dx.doi.org/10.4025/cienccuidsaude.v12 i2.19643

2. Carvalho KEG, Araújo EC. Educação em saúde para a promoção da saúde sexual de adolescentes: uma prática de enfermagem [Editorial]. Rev Enferm UFPE on line [Internet]. 2017. [citado em 16 set 2017]; 11(4). Disponível em: https://periodicos.ufpe.br/revistas/revistaenf ermagem/article/view/15222

3. Melo RHV, Felipe MCP, Cunha ATR, Vilar RLA, Pereira EJS, Carneiro NEA, et al. Roda de conversa: uma articulação solidária entre ensino, serviço e comunidade. Rev Bras Educ Méd. [Internet]. 2016 [citado em 23 set 2017]; 40(2):301-9.

DOI: http://dx.doi.org/10.1590/1981-

$52712015 \mathrm{v} 40 \mathrm{n} 2 \mathrm{e} 01692014$

4. Kovaleski DF, Zampieri MF, Zuco LP, Nitschke RG. Trajetória do Pró-PET - Saúde da família no cotidiano da promoção da saúde. Rev Bras Educ Méd. [Internet]. 2016 [citado em 25 set 2017]; 40(4):765-71.

DOI: http://dx.doi.org/10.1590/1981-

52712015v40n4e00422015

5. Silva MAM, Pinheiro AKB, Souza AMA, Moreira ACA. Promoção da saúde em ambientes hospitalares. Rev Bras Enferm. [Internet]. 2011 [citado em 12 fev 2018]; 64(3):596-9. DOI: $\quad$ http://dx.doi.org/10.1590/S003471672011000300027

6. Martins VF. Hospitais universitários federais e a nova reestruturação organizacional: o primeiro olhar, uma análise de um hospital universitário. Rev Adm Contab. [Internet]. 2011 [citado em 10 out 2017]; 3(2):4-22. Disponível em:

http://www.reacfat.com.br/index.php/reac/ar ticle/view/27

7. Cabral JR. Oficinas de educação em saúde com idosos: uma estratégia de promoção da qualidade de vida. Rev Enferm Digit Cuid Promoç Saúde [Internet]. 2015. [citado em 18 out 2017]; 1(2):71-5. DOI: http://www.dx.doi.org/10.5935/24465682.20150011

8. Ribeiro CRB, Sobóia VM. Popular education in health with fishermen: an experience outside the nurse's "comfort zone". Rev Pesqui Cuid Fundam. [Internet]. 2015 [citado em 13 mar 2018]; 7(3):2846-52. DOI: http://dx.doi.org/10.9789/2175-

5361.2015.v7i3.2846-2852

9. Silva KL, Araújo FL, Santos FBO, Andrade AM, Basílio NC, Sena RR. O que vem se falando por aí em competências no ensino da promoção da saúde na formação do enfermeiro? ABCS Health Sciences [Internet]. 2015[Citado em nov 2017]; 40(3):286-93. DOI: 10.7322/abcshs.v40i3.809 10. Saporetti GM, Miranda PSC, Belisário SA. 0 profissional de educação física e a promoção da saúde em núcleos de apoio à saúde da família. 
Trab Educ Saúde [Internet]. 2016 [citado em 11 set 2017]; 14(2):523-43. DOI: http://dx.doi.org/10.1590/1981-7746sip00113

11. Rosin SM, Gonçalves ACA, Hidalgo MM. Programa de educação tutorial: lutas e conquistas. ComInG [Internet]. 2017 [citado em 20 nov 2017]; 2(1):70-9. DOI: http://dx.doi.org/10.5902/2448190424495 12. Carvalho BC, Peduzzi M, Mandú ENT, Ayres JRCM. Trabalho e intersubjetividade: reflexão teórica sobre sua dialética no campo da saúde e enfermagem. Rev Latinoam Enferm. [Internet]. 2012 [citado em 22 set 2017]; 20(1):[8 telas]. DOI: $\quad$ http://dx.doi.org/10.1590/S010411692012000100004

13. Coriolano-Marinus MWV, Queiroga BAM, Moreno LR, Lima LS. Comunicação nas práticas em saúde: revisão integrativa da literatura. Saúde Soc. [Internet]. 2014 [citado em 22 set 2017]; 23(4):1356-69. DOI: http://dx.doi.org/10.1590/S0104-

12902014000400019

14. Almeida TJ, Salvador PTCO, Alves KYA, Sousa ICA. Playful education in health: report of an experience of "luminescent nurses". Rev Pesqui Cuid Fundam. [Internet]. 2013 [citado em 12 nov 2017]; 5(5):122-30. Disponível em: http://www.seer.unirio.br/index.php/cuidadof undamental/article/view/1642/pdf_1009

15. Pinto ACM, Oliveira IV, Santos ALS, Silva LES, Izidoro GSL, Mendonça RD et al. Percepção dos alunos de uma universidade pública sobre o
Programa de Educação pelo Trabalho para a saúde. Ciência \& Saúde Coletiva [Internet]. 2013 [citado em fev 2018]; 18(8):2201-10. DOI: http://dx.doi.org/10.1590/S1413-

81232013000800004

16. Pinto JMR. A teoria da ação comunicativa de Jürgen Habermas: conceitos básicos e possibilidades de aplicação à administração escolar. Paidéia (Ribeirão Preto) [Internet]. 1995 [citado em 12 set 2017]; 8-9:77-96. DOI: http://dx.doi.org/10.1590/S0103-

863X1995000100007

17. Ruiz MJF. A ação comunicativa na práxis pedagógica: um estudo a partir de Habermas. [dissertação]. Londrina: Universidade Estadual de Londrina; 2006. 106p.

18. Alves MA, Mello PL. Consciência social, comunidade ética e crítica da alienação em Habermas. Aufklärung, Rev Filos. [Internet]. 2016 [citado em 12 set 2017]; 3(2):101-12. DOI: https://doi.org/10.18012/arf.2016.30417

CONTRIBUIÇÕES
Nakita Maria Komori participou da
concepção, busca bibliográfica, redação, e
revisão crítica. Luiz Antonio Pertili
Rodrigues de Resende e Dalmo Correa
Filho atuaram na redação e revisão crítica.
Mirtes Helena dos Reis contribuiu na
redação e revisão crítica.

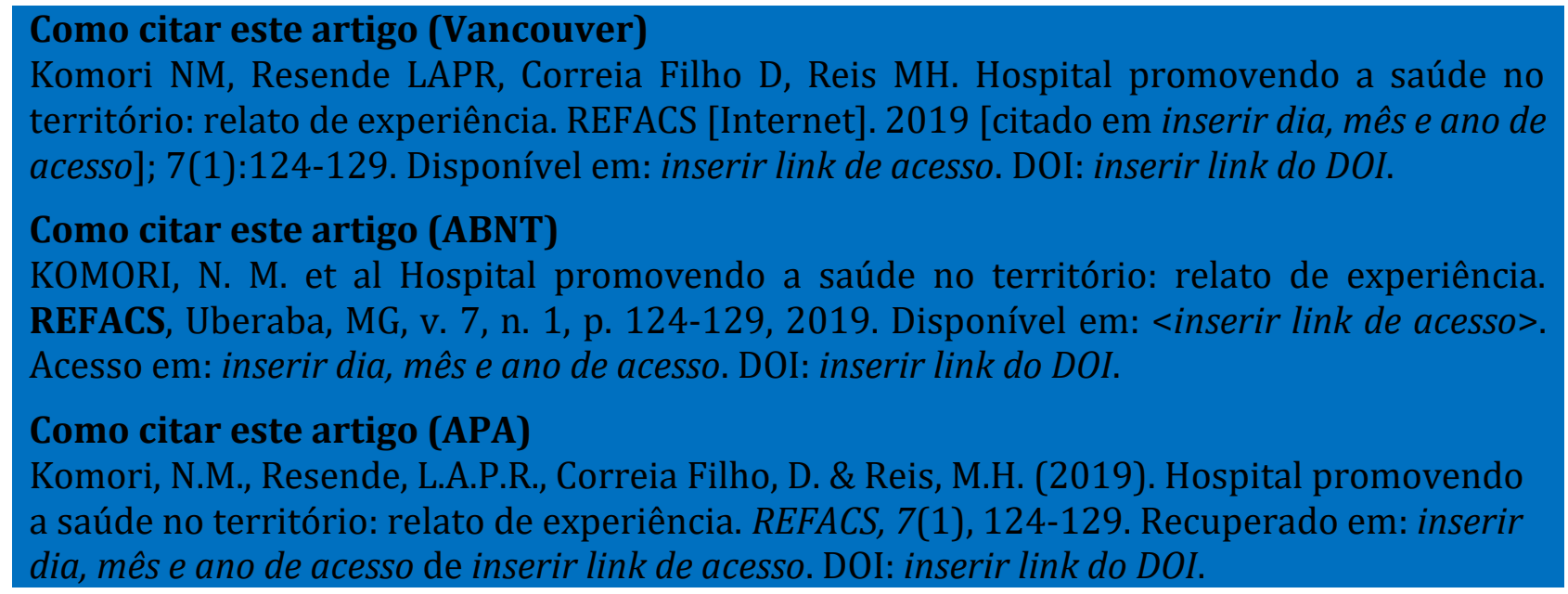

